\title{
ERGODIC RESULTS FOR CERTAIN CONTRACTIONS ON ORLICZ SPACES WITH FIXED POINTS
}

\author{
Diego Gallardo
}

\begin{abstract}
Let $\left(X, \mathcal{M}_{1} \mu\right)$ be a $\sigma$-finite measure space, $L_{\phi} \equiv L_{\phi}(X, \mathcal{M}, \mu)$ an Orlicz space associated to an $N$-function $\phi$ and let $T: L_{\phi} \longrightarrow L_{\phi}$ be a linear operator with a fixed point $h \neq 0$ a.e., such that

$$
\int_{X} \phi(|T f|) d \mu \leq \int_{X} \phi(|f|) d \mu \quad\left(f \in L_{\phi}\right)
$$

and it is either a \|\|$_{1}$-contraction in $L_{\phi} \cap L_{1}$ or a \|\|$_{\infty}$-contraction in $L_{\phi} \cap L_{\infty}$. The main result of this paper is that for a wide class of $N$. functions $\phi$, the ergodic maximal operator associated to $T$ is bounded in $L_{\phi}$. Moreover, for every $f \in L_{\phi}$ we have the almost everywhere convergence and the norm convergence of certain weighted averages which include the Césàro averages.
\end{abstract}

\section{Introduction and preliminaries}

Let $(X, \mathcal{M}, \mu)$ be a $\sigma$-finite measure space and $L_{\phi} \equiv L_{\phi}(X, \mathcal{M}, \mu)$ and Orlicz space associated to an $N$-function $\phi$ ( $L_{\phi}$ may be a complex Banach space). In this paper we will consider linear operators $T$ such that

i) $\int_{X} \phi(|T f|) d \mu \leq \int_{X} \phi(|f|) d \mu, f \in L_{\phi}$

ii) $T$ has a fixed point $h, h \neq 0$ a.e.

iii) $T$ is either a \|\|$_{1}$-contraction in $L_{\phi} \cap L_{1}$ or a \|\|$\|^{- \text {contraction in }}$ $L_{\phi} \cap L_{\infty}$.

The main aim of this paper is to prove that, for a wide class of $N$-functions $\phi$, the ergodic maximal operator $M_{T}$ defined by

$$
M_{T} f=\sup _{n \geq 1}\left|\frac{1}{n} \sum_{k=0}^{n-1} T^{k} f\right|
$$

Keywords: Almost everywhere convergence, Besicovitch sequences, Césàro-averages, Contractions, $\Delta_{2}$-condition, Ergodic maximal operator, Ergodic theorems, Extrapolation theorems, Fixed points, $N$-functions, norm convergence, Orlicz spaces, weighted averages. 1980 Mathematics subject classifications: Primary: 47A35. Secondary: 46 E30. 
is bounded in $L_{\phi}$ (dominated ergodic theorem). Moreover, we shall prove that if $\left\{b_{k}\right\}$ is a bounded Besicovitch sequence, then for every $f \in L_{\phi}$ there exists $f^{*} \in L_{\phi}$ such that

$$
\lim _{n \rightarrow \infty} \frac{1}{n} \sum_{k=0}^{n-1} b_{k} T^{k} f(x)=f^{*}(x) \quad \text { a.e., } \quad \lim _{n \rightarrow \infty}\left\|\frac{1}{n} \sum_{k=0}^{n-1} b_{k} T^{k} f-f^{*}\right\|_{(\phi)}=0 .
$$

A sequence of complex numbers $\left\{b_{k}\right\}$ is called a Besicovitch sequence if for every $\varepsilon>0$ there exists a trigonometric polynomial $\alpha_{\varepsilon}$ such that

$$
\limsup _{n \rightarrow \infty} \frac{1}{n} \sum_{k=0}^{n-1}\left|b_{k}-\alpha_{\varepsilon}(k)\right|<\varepsilon
$$

As a special case we obtain the almost everywhere convergence (individual ergodic theorem) and the norm convergence (mean ergodic theorem) of the Césàroaverages $n^{-1}\left(f+T f+\cdots+T^{n-1} f\right)$.

In the real $L_{p}$-case, with $1<p<\infty$, and $(X, \mathcal{M}, \mu)$ a finite measure space the corresponding dominated ergodic theorem is proved by A. de la Torre in [10]. R. Sato proved in [9] that the de la Torre's result may be extended to the case $(X, \mathcal{M}, \mu) \sigma$-finite and a complex $L_{p}$-space. The ergodic result for an operator which only satisfies conditions i) and iii) is an open problem even in the $L_{p}$-case, $1<p<\infty$.

The bounded Besicovitch sequences as weights in the averages were used by J.H. Olsen in [8].

In order to obtain the dominated ergodic theorem we first need some extrapolation theorems which extend the ones given by M.A. Akcoglu and R.V. Chacon in [1] and R. Sato in [9], for $L_{p}, 1<p<\infty$.

Now, we shall present the basic definitions and results concerning to $N$ functions and Orlicz spaces which will be used in this paper. The proofs of most of these results can be found in [5] or in II-13 of [7].

An $N$-function is a continuous and convex function $\phi:[0, \infty) \longrightarrow \mathbf{R}$ such that $\phi(s)>0, s>0, s^{-1} \phi(s) \longrightarrow 0$ as $s \longrightarrow 0$ and $s^{-1} \phi(s) \longrightarrow \infty$ as $s \longrightarrow \infty$.

The function $\phi$ is an $N$-function if and only if it has the representation $\phi(s)=\int_{0}^{s} \varphi$ where $\varphi:[0, \infty) \longrightarrow \mathbf{R}$ is continuous from the right, non decreasing such that $\varphi(s)>0, s>0, \varphi(0)=0$ and $\varphi(s) \longrightarrow \infty$ as $s \longrightarrow \infty$. More precisely $\varphi$ is the right derivate of $\phi$ and will be called the density function of $\phi$.

Associated to $\varphi$ we have the function $\rho:[0, \infty) \longrightarrow \mathbf{R}$ defined by $\rho(t)=$ $\sup \{s: \varphi(s) \leq t\}$ which has the same aforementioned properties of $\varphi$. We will call $\rho$ the generalized inverse of $\varphi$.

The $N$-function $\psi$ defined by $\psi(t)=\int_{0}^{t} \rho$ is called the complementary $N$ function of $\phi$. Thus, if $\phi(s)=p^{-1} s^{p}, p>1$, then $\psi(t)=q^{-1} t^{q}$ where $p q=p+q$. 
Young's inequality asserts that $s t \leq \phi(s)+\psi(t)$ for $s, t \geq 0$, equality holding if and only if $\varphi(s-) \leq t \leq \varphi(s)$ or else $\rho(t-) \leq s \leq \rho(t)$ (See [3]).

If $\phi_{1}$ and $\phi_{2}$ are $N$-functions with complementary $N$-functions given by $\psi_{1}$ and $\psi_{2}$ respectively, then, the inequality for complementary functions asserts that if $\phi_{1}(s) \leq \phi_{2}(s)$ for $s \geq s_{0}$, then $\psi_{2}(t) \leq \psi_{1}(t)$ for $t \geq \varphi_{2}\left(s_{0}\right)$, where $\varphi_{2}$ is the density function of $\phi_{2}$.

An $N$-function $\phi$ is said to satisfy the $\triangle_{2}$-condition in $\left[s_{0}, \infty\right), s_{0} \geq 0$, if there exists a constant $\alpha$ such that $\phi(2 s) \leq \alpha \phi(s)$ for every $s \geq s_{0}$.

If $\varphi$ is the density function of $\phi$, then $\phi$ satisfies $\Delta_{2}$ in $\left[s_{0}, \infty\right)$ if and only if there exists a constant $\alpha>1$ such that $s \varphi(s) \leq \alpha \phi(s), s \geq s_{0}$.

The $\triangle_{2}$-condition for $\phi$ does not transfer necessarily to the complementary $N$-function.

If $(X, \mathcal{M}, \mu)$ is a $\sigma$-finite measure space we denote by $\mathbf{M}=\mathbf{M}(X, \mathcal{M}, \mu)$ the space of $\mathcal{M}$-measurable and $\mu$-a.e. finite functions from $X$ ro $\mathrm{R}$ or to C. If $\phi$ is an $N$-function we consider the Orlicz spaces $L_{\phi} \equiv L_{\phi}(X, \mathcal{M}, \mu)$ and $L_{\phi}{ }^{*} \equiv L_{\phi}{ }^{*}(X, \mathcal{M}, \mu)$ defined by $L_{\phi}=\left\{f \in \mathbf{M}: \int_{X} \phi(|f|) d \mu<\infty\right\}$ and $L_{\phi}{ }^{*}=\left\{f \in \mathbf{M}: f g \in L_{1}\right.$ for all $\left.g \in L_{\psi}\right\}$ where $\psi$ is the complementary $N$-function of $\phi$. We have $L_{\phi} \subset L_{\phi}^{*}$ and if $\phi$ satisfies $\Delta_{2}$ then $L_{\phi}=L_{\phi}{ }^{*}$.

We have that $L_{\phi}^{*}$ is a linear space with the usual operations on which we may define the norms $\|f\|_{\phi}=\sup \left\{\int_{X}|f g| d \mu: g \in S_{\psi}\right\}$, where $S_{\psi}=\{g \in$ $\left.L_{\psi}: \int_{X} \psi(|g|) d \mu \leq 1\right\}$, and $\|f\|_{(\phi)}=$ inf $\left\{\lambda>0: \int_{X} \phi\left(\lambda^{-1}|f|\right) d \mu \leq 1\right\}$ which are called Orlicz norm and Luxemburg norm respectively. Both norms are equivalent.

Holder's inequality asserts that for every $f \in L_{\phi}{ }^{*}$ and cvery $g \in L_{\psi}{ }^{*}$ we have $\|f g\|_{1} \leq\|f\|_{(\phi)}\|g\|_{\psi}$ where $\phi$ and $\psi$ are complementary $N$-functions.

If $\phi(s)=s^{p}$ with $p>1$ then $L_{\phi}{ }^{*}=L_{\phi}=L_{p},\|f\|_{(\phi)}=\|f\|_{p}$ and $\|g\|_{\psi}=\|g\|_{q}$ where $p q=p+q$.

The convergence $f_{n} \longrightarrow f$ in $\left.\left.\left[L_{\phi}{ }^{*},\right]\right] \quad \|_{\phi}\right]$ implies the mean convergence $\lim _{n \rightarrow \infty} \int_{X}\left(\left|f_{n}-f\right|\right) d \mu=0$ but, in general, mean convergence only implies norm convergence when $\phi$ satisfies $\Delta_{2}$. Then the set $S$ of simple functions (with support of finite measure) is dense in $\left[L_{\phi},\|\|_{\phi}\right]$ if $\phi$ satisfies $\triangle_{2}$.

If $\phi$ verifies $\Delta_{2}$, then for every continuous linear functional $F$ over $\left[L_{\phi},\|\|_{(\phi)}\right]$ there exists an unique function $g \in L_{\psi}{ }^{*}$ such that $F(f)=\int_{X} f g d \mu, f \in L_{\phi}$, and moreover $\|F\|_{(\phi)}=\|g\|_{\psi}$, where $\psi$ is the complementary $N$-function of $\phi$, but if $\phi$ does not satisfy $\triangle_{2}$ then there exist linear functionals on $L_{\phi}{ }^{*}$ which are not represented by functions of $L_{\psi}{ }^{*}$.

If $\phi$ and $\psi$ satisfy $\triangle_{2}$ then $\left[L_{\phi},\|\|_{(\phi)}\right]$ is reflexive.

In the following, we shall always assume that $(X, \mathcal{M}, \mu)$ is a $\sigma$-finite measure space and $\phi$, together with its complementary $N$-function $\psi$, satisfy the $\triangle_{2-}$ condition in $[0, \infty)$. The $\Delta_{2}$-condition for $\phi$ is a very important condition that plays fundamental roles in many questions and the best known Orlicz spaces are associated to functions which satisfy $\triangle_{2}$. The $\triangle_{2}$-condition for $\psi$ may seem 
to be a restrictive assumption. Some know Orlicz spaces as, for example, the Zygmund Orlicz space $L \log L$ and the $L \log ^{k} L$ spaces, $k>0$, are associated to $N$-functions which satisfy $\triangle_{2}$ but their complementary $N$-functions do not; but the above spaces do not satisfy our dominated ergodic result. In fact the $\triangle_{2}$-condition for the complementary $N$-function is necessary for such result.

Precisely, let $([0,1], \mathcal{B}, \lambda)$ be the Lebesgue-space and let $\tau$ an invertible $\lambda$ measure preserving transformation from $[0,1]$ into itself. In [2] B. Bru and H. Heinich characterize the Orlicz spaces, associated to Young's functions, for which the ergodic maximal operator associated to the operator $T$, defined by $T f=f \circ \tau^{-1}$, is bounded in $L_{\phi}$ (classical dominated ergodic theorem) (the Young's functions in [2] are our $N$-functions). The characterizing condition given in [2] is the condition of comoderation on $\phi$.

The function $\phi$ is said to be comoderated if there exist $s_{0}$, a and $b>1$ such that $\varphi(a s) \geq b \varphi(s)$ for $s \geq s_{0}$, where $\varphi$ is the density function of $\phi$ or, equivalently, if there exist $s_{0}, a$ and $b>1$ such that $\phi(a s) \geq a b \phi(s)$ for $s \geq s_{0}$ (in [2] a function continuous from the left is taken as density function of $\phi$ whereas our density function is right continuous).

The paper [2] does not establisch the equivalence between the comoderation of $\phi$ and the moderation $\left(\triangle_{2}\right.$-condition in some $\left.\left[t_{0}, \infty\right)\right)$ of the complementary $N$-function $\psi$ unless $\varphi$ be continuous. However, we observe that the comoderation of $\phi$ is equivalent to the moderation of $\psi$. At the same time, we shall prove another characterization of the moderation of $\psi$, which is used in this paper, and which appear in [2], [5] and in the rest of the literature with more. restrictive hypothesis. Exactly:

Proposition 1.2, Let $\phi$ be an $N$-function and $\psi$ the complementary $N$ function of $\phi$. The following conditions are equivalent:

a) $\phi$ is comoderated.

b) $\psi$ is moderated.

c) There exist $s_{0}$ and $\beta>1$ such that $\beta \phi(s) \leq s \varphi(s)$ for $s \geq s_{0}$.

Proof: $a) \Longrightarrow b$ ). If $\phi$ is comoderated then $\phi(s) \leq \phi_{1}(s)$ for $s \geq s_{0}$ where $\phi_{1}$ is the $N$-function given by $\phi_{1}(s)=(a b)^{-1} \phi(a s)$. The complementary function of $\phi_{1}$ is given by $\psi_{1}(t)=(a b)^{-1} \psi(b t)$. Taking into account the inequality for complementary $N$-functions we obtain that $\psi(b t) \leq a b \psi(t)$ for $t \geq t_{0}=\varphi_{1}\left(s_{0}\right)$, where $b>1$, which equivales to condition $\Delta_{2}$ of $\psi$ for $t \geq t_{0}$.

$b) \Longrightarrow c$ ). Let $\rho$ be the generalized-inverse of $\varphi$. Since $\psi$ is moderated there exist $t_{0}$ and $\alpha>1$ such that $t \rho(t) \leq \alpha \psi(t)$ for every $t \geq t_{0}$. On the other hand, it follows from the equality cases in Young's inequality that $t \rho(t)=$ $\phi(\rho(t))+\psi(t)$ and therefore

$$
\phi(\rho(t)) \leq \alpha^{-1}(\alpha-1) t \rho(t), \quad t \geq t_{0} .
$$

Then, since $\rho(\varphi(s)) \geq s$ and the function $u \longrightarrow u^{-1} \phi(u)$ increases for $u>0$ we obtain

$$
s^{-1} \phi(s) \leq \phi(\rho(\varphi(s))) / \rho(\varphi(s)) \leq \alpha^{-1}(\alpha-1) \varphi(s), \quad s \geq \rho\left(t_{0}\right)
$$


and thus we obtain c) with $s_{0}=\rho\left(t_{0}\right)$ and $\beta=\alpha(\alpha-1)^{-1}>1$.

$c) \Longrightarrow a$ ). Condition c) implies that there exist $s_{0}$ and $\beta>1$ such that the function $s \longrightarrow s^{-\beta} \phi(s)$ increases for $s \geq s_{0}$ (or for $s>s_{0}$ if $s_{0}=0$ ). Then, if $a>1$ is such that $a^{\beta-1} \geq 2$ we have $\phi(a s) \geq a^{\beta} \phi(s) \geq 2 a \phi(s)$ for $s \geq s_{0}$ and thus we obtain the comoderation of $\phi$.

Note. Since $\varphi(0)=\rho(0)=0$, if some of the conditions of Proposition 1.2 is satisfied for every $s \geq 0$, then the others two conditions are also valids for every $s \geq 0$.

In this way, the moderation of $\psi$ is necessary for the classical dominated ergodic result and, therefore, for our dominated ergodic result since that the operator $T$, defined by $T f=f \circ \tau^{-1}$ satisfies conditions i), ii) and iii), whatever the $N$-function $\phi$ may be. On the other hand, the space $([0,1], \mathcal{B}, \lambda)$ is of finite measure and our spaces can be of infinite measure. For this reason we shall assume the $\triangle_{2}$-condition in $[0, \infty)$, but un the case $\mu(X)<\infty$ the argument which we shall use can be adapted if only we suppose the $\Delta_{2}$-condition in some $\left[s_{0}, \infty\right)$.

Our results are valid, for example, for the known $L^{p} \log ^{k} L$ spaces, with $p>1$ and $k \geq 0$, since the $N$-functions of the form $\phi(s)=s^{p} \log ^{k}(1+s)$ satisfy that $1<p<\phi(s) / s \varphi(s) \leq p+b$ for every $s>0$ and certain constant $b$.

\section{Extrapolation Theorems}

We first observe that the convexity theorem for positive operators given by M.A. Akcoglu and R.V. Chacon in [1] can be easily extended to Orlicz spaces, following the same type of arguments, as follows

Proposition 2.1. Let $\phi$ be an $N$-function strictly convex in some interval and let $T$ be a conservative positive contraction in $L_{1}$ such that

$$
\int_{X} \phi(|T f|) d \mu \leq \int_{X} \phi(|f|) d \mu, \quad\left(f \in L_{1} \cap L_{\phi}\right) .
$$

Then, $\|T f\|_{\infty} \leq\|f\|_{\infty}$ for every $f \in L_{1} \cap L_{\infty}$.

Proof: The operator $T$ is said to be conservative when $\mu(D)=0$, where $D$ is the dissipative part of $X$ with respect to $T$.

First assume that $\mu(X)<\infty$. It is enough to prove that $T c \leq c$ almost everywhere for some constant $c \neq 0$.

We have that $\varphi$ increases strictly in some interval $I$, where $\varphi$ is the density function of $\phi$. Let $c \in I$ with $c \neq 0$. Then, we get that

$$
\phi(c+s)>\phi(c)+s \varphi(c) \quad(0 \neq s \geq-c) .
$$

Since $T$ is conservative we have $\int_{X} T f d \mu=\int_{X} f d \mu$ for every $f \in L_{1}$. 
Let $T c(x)=c+g(x)$; then $\int_{X} g d \mu=0$ and therefore if $\mu\{x \in X$ : $g(x)>0\}>0$ we have

$$
\int_{X} \phi(|T c|) d \mu>\int_{X} \phi(c) d \mu
$$

which contradicts (2.2). This proves that $T c \leq c$.

The general case follows from the preceding by a method similar to the one given in [1] using the following result:

Lemma 2.4. Let $\phi$ be an $N$-function and $T$ a positive contraction in $L_{1}$ satisfing (2.2). Then, for every $A \in M$ there exists a linear operator

$T_{A}: L_{1}(A) \longrightarrow L_{1}(A)$ such that

a) $T_{A}$ is a positive contraction in $L_{1}(A)$ and

$$
\int_{X} \phi\left(\left|T_{A} f\right|\right) d \mu \leq \int_{X} \phi(|f|) d \mu,\left(f \in L_{1}(A) \cap L_{\phi}(A)\right) .
$$

b) For every $f \in L_{1}{ }^{+}(A)$ and every $n \geq 1$

$$
\sum_{k=0}^{n} T^{k} f(x) \leq \sum_{k=0}^{n} T_{A}^{k} f(x) \quad \text { a.e. in } A \text {. }
$$

The proof of Lemma 2.4 can be obtained easily following the arguments of [1].

\section{Remarks,}

1. The conservative condition of $T$ cannot be eliminated from the hypothesis of Proposition 2.1 since in $R$ with Lebesgue-measure if $T f(x)=\sqrt{2} f(2 x)$ then $T$ is a positive contraction in $L_{1}$, an isometry in $L_{2}$ but $\|T f\|_{\infty}=$ $\sqrt{2}\|f\|_{\infty}$.

2. There exist $N$-function which are strictly convex over no interval. An example is the following. We consider the dyadic intervals $I_{n}=\left[2^{n-1}, 2^{n}\right)$ and $J_{n}=\left[2^{-n}, 2^{-n+1}\right)$ where $n$ is a positive integer and let $\varphi:[0, \infty) \longrightarrow[0, \infty)$ bc defined by $\varphi(0)=0, \varphi(t)=2^{-n}$ if $t \in J_{n}$ and $\varphi(t)=2^{n-1}$ if $t \in I_{n}$. Then $\phi$ defined by $\phi(s)=\int_{0}^{s} \varphi$ is an $N$-function. Since $\phi(2 s)=4 \phi(s)$ we have that $\phi$, as well as its complementary $N$-function, satisfy the $\triangle_{2}$-condition. However $\phi$ is not strictly convex over any interval. Furthemore there is no constant $c \neq 0$ such that (2.3) holds.

However most of $N$-functions are strictly convex in some interval.

In the following results the operators are not necessarily positive but they have a fixed point $h$ with $h \neq 0$ a.e. 
Theorem 2.5. Let $\phi$ be an $N$-function, strictly convex in some interval and let $T: L_{\phi} \longrightarrow L_{\phi}$ be a linear operator such that

i) $\int_{X} \phi(|T f|) d \mu \leq \int_{X} \phi(|f|) d \mu, \quad\left(f \in L_{\phi}\right)$.

ii) $\|T f\|_{1} \leq\|f\|_{1}, \quad\left(f \in L_{1} \cap L_{\phi}\right)$.

izi) There exists $h \in L_{\phi}, h \neq 0$ a.e., such that $T h=h$.

Then, $\|T f\|_{\infty} \leq\|f\|_{\infty}$ for every $f \in L_{1} \cap L_{\infty}$ and consequently for every $f \in L_{\phi} \cap L_{\infty}$.

Proof: In this proof we follows the idea given by Sato in [9].

Let $k$ be such that $\phi(s)<s$ for $0<s<k$. Given $f \in L_{1} \cap L_{\infty}$ let $B=\{x \in X:|f(x)| \geq k\}$; then $\mu(B)<\infty$ and therefore $\int_{X} \phi(|f|) d \mu \leq$ $\|f\|_{1}+\mu(B) \phi(\|f\|)<\infty$. Consequently $L_{1} \cap L_{\infty} \subset L_{\phi}$.

Let $\hat{T}: L_{1} \longrightarrow L_{1}$ be the linear extension of $T:\left[L_{1} \cap L_{\phi},\|\|_{\mathrm{I}}\right] \rightarrow L_{1}$ and $P$ the linear modulus of $\hat{T}$. (See Theorem 4.1.1 in [6]). We shall prove that $P$ satisfies the hypotheses of Proposition 2.1 and therefore $\|P f\|_{\infty} \leq\|f\|_{\infty}$ $f \in L_{1} \cap L_{\infty}$; in this way, since $|\hat{T} f| \leq P|f|, f \in L_{1}$, and $L_{1} \cap L_{\infty} \subset L_{1} \cap L_{\phi}$ we obtain that $\|T f\|_{\infty} \leq\|f\|_{\infty}, f \in L_{1} \cap L_{\infty}$, and consequently for every $f \in L_{\phi} \cap L_{\infty}$ since $L_{1} \cap L_{\infty}$ is dense in $L_{\phi} \cap L_{\infty}$ with the $L_{\infty}$-norm.

Now, we show that $P$ satisfies the conditions of Proposition 2.1. The $\triangle_{2}$ condition implies that $L_{1} \cap L_{\phi}$ is dense in $\left[L_{\phi},\left\|^{\prime}\right\|_{(\phi)}\right]$. On the other hand, it follows from i) that $\|T f\|_{\{\phi)} \leq\|f\|_{(\phi)}, f \in L_{\phi}$, and consequently given $\varepsilon>0$ there is $f_{\epsilon} \in L_{1} \cap L_{\phi}$ such that for every $n \geq 1$

$$
\left\|h-\frac{I}{n} \sum_{k=0}^{n-1} T^{k} f_{\varepsilon}\right\|_{(\phi)} \leq \varepsilon / 2 .
$$

If $T$ is a power bounded linear operator in a reflexive Banach space $V$, that is, the powers $T^{k}, k \geq 0$, are uniformly bounded in $V$, then the Césàro-averages.

$$
R_{n} f=\frac{1}{n} \sum_{k=0}^{n-1} T^{k} f
$$

converge in norm to a $T$-invariant limit for all $f \in V$ (See Theorem 2.1.2 in $[6])$.

Lef $f_{\varepsilon}{ }^{*}$ be the limit in $\left[L_{\phi},\|\|_{(\phi)}\right]$ of $R_{n} f_{\varepsilon}$. It follows from (2.6) that for $0<\varepsilon<1$ we have $\left\|h-f_{\epsilon}^{*}\right\|_{(\phi)}<\varepsilon$ and consequently

$$
\int_{X} \phi\left(\left[h-f_{\epsilon}^{*} \mid\right) d \mu<\varepsilon\right.
$$

On the other hand, $f_{e}^{*}(x)=0$ for a.e. $x \in D$, where $D$ is the dissipative part of $X$ with respect to $P$, since (Theorem 3.1 .6 in [6]) $\sum_{k \geq 0} P^{k} f(x)<\infty$ 
on $D$ for all $f \in L_{1}{ }^{+}$. Since $\phi(|h|)>0$ a.e. (2.7) shows that $\mu(D)=0$ and thus $P$ is conservative.

Now, in order to prove that $P$ satisfies condition (2.2) we consider the Akcoglu and Brunel's theorem related with the structure of $\hat{T}$ on the conservative part $C$ of $X$ with respect to $P$ (see Theorem 4.1 .10 in [6]). Let $\mathcal{F}$ be the family of $P$-absorbing subsets of $C$; there exists a set $\Gamma \in \mathcal{F}$ and a function $s \in L_{\infty}(\Gamma)$, with $|s|=1$ on $\Gamma$, such that $\hat{T} f=\bar{s} P(s f)$ for any $f \in L_{1}(\Gamma)$, where $\bar{s}$ is the complex conjugate of $s$, and if $\triangle=C-\Gamma$ then $(I-T) L_{1}(\Delta)$ is dense in $L_{1}(\Delta)$.

We have that $\operatorname{supp} T\left(\chi_{\Gamma} h\right) \subset \Gamma$ and $\operatorname{supp} T(\chi \Delta h) \subset \Delta ;$ therefore $T g=g$ where $g=\chi_{\Delta} h$. Carryng out a similar reasoning to the used for $h$ we have that for every $\varepsilon>0$ there exist $f_{\varepsilon} \in L_{1}(\Delta) \cap L_{\phi}(\Delta)$ and $f_{\varepsilon}^{*} \in L_{\phi}(\Delta)$ such that $\left\|g-f_{\varepsilon}{ }^{*}\right\|_{(\phi)}<\varepsilon$ and $\lim _{n \rightarrow \infty}\left\|R_{n} f_{\varepsilon}-f_{\varepsilon}{ }^{*}\right\|_{(\phi)}=0$.

Given $\eta>0$ there is $u_{\eta} \in L_{1}(\Delta)$ such that $\left\|u_{\eta}-T u_{\eta}-f_{\varepsilon}\right\|_{1}<\eta / 2$ and therefore for every $n \geq 1$ we have $\left\|n^{-1}\left(u_{\eta}-T^{n} u_{\eta}\right)-R_{n} f_{\epsilon}\right\|_{1}=\| R_{n}\left(u_{\eta}-\right.$ $\left.T u_{\eta}-f_{\epsilon}\right) \|_{1}<\eta / 2$, which proves that $\lim _{n \rightarrow \infty}\left\|R_{n} f_{\varepsilon}\right\|_{i}=0$ and so $f_{\varepsilon}{ }^{*}(x)=$ 0 a.e. This shows that $\|g\|_{(\phi)}=0$ and conseguently $\mu(\Delta)=0$. Then, we have $\hat{T} f=\bar{s} P(s f)$ for every $f \in L_{1}$ and therefore it follows from i) that $\int_{X} \phi(|P f|) d \mu=\int_{X} \phi(|\bar{s} \hat{T}(\bar{s} f)|) d \mu \leq \int_{X} \phi(|f|) d \mu$ for every $f \in L_{1} \cap L_{\phi}$ and this finishes the proof.

Now, our aim is to prove that the roles of $L_{\mathrm{1}}$ and $L_{\infty}$ in Theorem 2.5 can be interchanged. For this we shall considerer the adjoint operator of $T$.

Let $T: L_{\phi} \longrightarrow L_{\phi}$ be a bounded linear operator; more precisely, we suppose that there is a constant $C$ such that $\|T f\|_{(\phi)} \leq C\|f\|_{(\phi)}, f \in L_{\phi}$. Then, if $g \in L_{\psi}{ }^{*}$, where $\psi$ is the complementary $N$-function of $\phi$, the linear functional $F$ over $\left[L_{\phi},\|\|_{(\phi)}\right]$ defined by $F(f)=\int_{X} g T f d \mu$ is continuous since by Holder's inequality we have $\left\{F(f) \mid \leq C\|g\|_{\psi}\|f\|_{(\phi)}\right.$ and therefore, since $\phi$ satisfies $\Delta_{2}$, there exists an unique function $g^{*} \in L_{\psi}{ }^{*}$ such that $\int_{X} g T f d \mu=\int_{X} f g^{*} d \mu, f \in$ $L_{\phi}$. Then, we can define the bounded linear operator $T^{*}: L_{\psi}{ }^{*} \longrightarrow L_{\psi}{ }^{*}, g \longrightarrow$ $T^{*} g$, where $T^{*} g$ is the function in $L_{\psi}^{*}$ such that

$$
\int_{x} g T f d \mu=\int_{X} f T^{*} g d \mu, \quad f \in L_{\phi}
$$

We shall call $T^{*}$ the adjoint operator of $T . T^{*}$ satisfies $\left\|T^{*} g\right\|_{\psi} \leq C\|g\|_{\psi}$. In our case we have

Lemma 2.8. Let $T: L_{\phi} \rightarrow L_{\phi}$ be a linear operator such that

$$
\int_{X} \phi(|T f|) d \mu \leq \int_{X} \phi(|f|) d \mu \quad\left(f \in L_{\phi}\right)
$$

Then, its adjoint operator $T^{*}$ satisfies

$$
\int_{X} \psi\left(\left|T^{*} g\right|\right) d \mu \leq \int_{X} \psi(|g|) d \mu \quad\left(g \in L_{\psi}\right)
$$


and moreover, if $T$ admits an invariant function $h$ with $h \neq 0$ a.e., then there exists $g \in L_{\psi}$ with $g \neq 0$ a.e., such that $T^{*} g=g$.

Proof: We write sig $z$ for $z / \mid z \uparrow$ and by $\tilde{u}$ we denote the complex conjugate of u. For $g \in L_{\phi}{ }^{+}$we have

$$
\begin{aligned}
\int_{X} f\left|T^{*} g\right| d \mu=\left|\int_{X} f\left(\operatorname{sig} \overline{T^{*} g}\right) T^{*} g d \mu\right| & \leq \int_{X}\left|T\left(f \operatorname{sig} \overline{T^{*} g}\right)\right||g| d \mu \leq \\
& \leq \int_{X} \phi(f) d \mu+\int_{X} \psi(|g|) d \mu .
\end{aligned}
$$

Let $\varphi$ be the density function of $\phi$ and $\rho$ the generalized inverse of $\varphi$. Since $\psi$ satisfies $\triangle_{2}$ there exists $\alpha>1$ such that $s \rho(s) \leq \alpha \psi(s)$ and therefore $\phi(\rho(s))=$ $s \rho(s)-\psi(s) \leq(\alpha-1) \psi(s)$. Therefore, for every $g \in L_{\psi}$ the function $\rho\left(\left|T^{*} g\right|\right)$ belongs to $L_{\phi}{ }^{+}$and so (2.9) follows from (2.10) for $f=\rho\left(\left|T^{*} g\right|\right)$.

Now, let us assume that $T h=h$ with $h \neq 0$ a.e. If $\varphi$ is not continuous then there exists an at most countable set of positive reals $s_{1}, s_{2}, \ldots, s_{n}$ where $\varphi$ is not continuous; in this situation, since $h \in L_{\phi}$, it is easy to see that $\left\{c>0: \mu\left\{x \in X:\left|s_{i}^{-1} h(x)\right|=c\right\}>0\right\}$ is at most countable and therefore there exists $\lambda>0$ such that for every $s_{i}$ we have

$$
\mu\left\{x \in X:\left|\lambda^{-1} h(x)\right|=s_{i}\right\}=0 .
$$

In the case $\varphi$ continuous (2.11) holds trivially with $\lambda=1$.

Let $u=\lambda^{-1} h$ and $g=\varphi(|u|) \operatorname{sig} \bar{u}$. We have that $g \neq 0$ a.e. and $g \in L_{\phi}$ since $\phi$ satisfies $\triangle_{2}$. It follows from (2.9) that

$$
\begin{aligned}
& \int_{X}|u| \varphi(|u|) d \mu=\left|\int_{X} u T^{*} g d \mu\right| \leq \int_{X}|u|\left\{T^{*} g \mid d \mu \leq \int_{X} \phi(|u|) d \mu+\right. \\
& +\int_{X} \psi\left(\left|T^{*} g\right|\right) d \mu \leq \int_{X} \phi(|u|) d \mu+\int_{X} \psi(\varphi(|u|)) d \mu=\int_{X}|u| \varphi(|u|) d \mu
\end{aligned}
$$

and therefore

$$
\int_{X}|u|\left|T^{*} g\right| d \mu=\int_{X}\left(\phi(|u|)+\psi\left(\left|T^{*} g\right|\right)\right) d \mu .
$$

Then, Young's inequality shows that

$$
\left|u T^{*} g\right|=\phi(|u|)+\psi\left(\left|T^{*} g\right|\right) \quad \text { a.e. }
$$

It follows from (2.11) and (2.13) that $\left|T^{*} g\right|=\varphi(|u|)$ a.e. On the other hand we obtain from (2.12) that (sig $\bar{u}) \operatorname{sig} \overline{T^{*} u}=1$ and therefore $T^{*} g=g$ which finishes the proof of the Lemma.

Theorem 2.5 and Lemma 2.8 imply easy 
Theorem 2.14. Let $\phi$ be an $N$-function whose complementary $N$-function is strictly convex in some interval and let $T: L_{\phi} \longrightarrow L_{\phi}$ be a linear operator such that

i) $\int_{X} \phi(|T f|) d \mu \leq \int_{X} \phi(|f|) d \mu, \quad\left(f \in L_{\phi}\right)$.

ii) $\|T f\|_{\infty} \leq\|f\|_{\infty}, \quad\left(f \in L_{\infty} \cap L_{\phi}\right)$.

iii) There exists $h \in L_{\phi}, h \neq 0$ a.e., such that $T h=h$.

Then, $\|T f\|_{1} \leq\|f\|_{1}$ for every $f \in L_{1} \cap L_{\phi}$.

Proof: Let $\psi$ be the complementary $N$-function of $\phi, T^{*}$ the adjoint operator of $T$ and let $\left\{A_{n}\right\}$ be an increasing sequence of measurable sets with $\mu\left(A_{n}\right)<\infty$ and $X=\cup A_{n}$. Then, for every $g \in L_{1} \cap L_{\psi}$ we have

$$
\int_{X}\left|T^{*} g\right| d \mu=\lim _{n \rightarrow \infty}\left|\int_{X} g T\left(\chi_{A_{n}} \operatorname{sig} \overline{T^{*} g}\right) d \mu\right| \leq\|g\|_{1} .
$$

Consequently, $\left\|T^{*} g\right\|_{\infty} \leq\|g\|_{\infty}$ for every $g \in L_{\psi} \cap L_{\infty}$ and therefore for any $f \in L_{1} \cap L_{\phi}$ and $n \geq 1$ we get $\left|\int_{X} f T^{*}\left(\chi_{A_{n}} \operatorname{sig} \overline{T f}\right) d \mu\right| \leq\|f\|_{1}$ and thus $\|T f\|_{1} \leq\|f\|_{1}$.

\section{Ergodic results}

Theorem 3.1. (Dominated, individual and mean weighted ergodic theorem). Let $\phi$ and $T$ satisfy the hypotheses of the extrapolation theorem 2.5 or 2.14 . Then

a) The ergodic maximal operator $M_{T}$-defined by (1.1) is bounded in $\left[L_{\phi},\|\|_{(\phi)}\right]$.

b). If $\left\{b_{k}\right\}$ is a bounded Besicovitch sequence, then for every $f \in L_{\phi}$ there exists $f^{*} \in L_{\phi}$ such that

$$
\lim _{n \rightarrow \infty} \frac{1}{n} \sum_{k=0}^{n-1} b_{k} T^{k} f(x)=f^{*}(x) \quad \text { a.e., } \quad \lim _{n \rightarrow \infty}\left\|\frac{1}{n} \sum_{k=0}^{n-1} b_{k} T^{k} f-f^{*}\right\|_{(\phi)}=0 .
$$

Proof: Since $L_{1} \cap L_{\infty} \subset L_{\phi}$ it follows from Theorem 2.5 or 2.14 that $T: L_{1} \cap$ $L_{\phi} \longrightarrow L_{1}$ admits an unique extension $\hat{T}: L_{1} \longrightarrow L_{1}$ which is a DunfordSchwartz operator, that is, $\|\tilde{T} f\|_{1} \leq\|f\|_{1}, f \in L_{1}$, and $\|\hat{T} f\|_{\infty} \leq\|f\|_{\infty}$, $f \in L_{1} \cap L_{\infty}$. Therefore the linear modulus $P$ of $\hat{T}$ is also a Dunfort-Schwartz operator.

Consequently, for every $f \in L_{\mathrm{I}}$ and $\lambda>0$ we have (see Theorem 2.3 .2 in [4])

$$
\mu\left\{x \in X: M_{P} f(x)>\lambda\right\} \leq \lambda^{-1} \int_{X}|f| d \mu,
$$

where $M_{P}$ is the maximal operator associated to $P$. Moreover, trivially, $\left\|M_{P} f\right\|_{\infty} \leq\|f\|_{\infty}$ for $f \in L_{1} \cap L_{\infty}$. 
For $f \in L_{1} \cap L_{\phi}$ set $f_{\lambda}=f \chi_{A(\lambda)}$ and $f^{\lambda}=f-f_{\lambda}$ where $A(\lambda)=\{x \in X$ : $|f(x)|>\lambda / 2\}$. We have $f_{\lambda} \in L_{1}, f^{\lambda} \in L_{1} \cap L_{\infty}$ and therefore

$$
\int_{X} \phi\left(M_{P} f\right) d \mu=\int_{0}^{\infty} \varphi(\lambda) \mu\left\{x \in X: M_{P} f(x)>\lambda\right\} d \lambda \leq
$$

$$
\leq 2 \int_{0}^{\infty} \lambda^{-1} \varphi(\lambda)\left(\int_{X}\left|f_{\lambda}\right| d \mu\right) d \lambda=2 \int_{X}|f(x)|\left(\int_{0}^{2|f(x)|} \lambda^{-1} \varphi(\lambda) d \lambda\right) d \mu(x),
$$

where $\varphi$ is the density function of $\phi$.

Integrating by parts, we obtain

$$
\int_{0}^{s} \lambda^{-1} \varphi(\lambda) d \lambda=s^{-1} \phi(s)+\int_{0}^{s} \lambda^{-2} \phi(\lambda) d \lambda \quad, \quad(s>0) .
$$

Since the $N$-function complementary of $\phi$ satisfies $\Delta_{2}$ there exists a constant $\beta>1$ such that $\beta \phi(s) \leq s \varphi(s), s \geq 0$; then, if $0<\lambda<1$ we have that $\lambda^{-2} \phi(\lambda) \leq \phi(1) \lambda^{\beta-2}$ and therefore $\int_{(0,3]} \lambda^{-2} \phi(\lambda) d \lambda<\infty$. Then, (3.3) shows that

$$
\int_{0}^{s} \lambda^{-1} \varphi(\lambda) d \lambda<\beta(\beta-1)^{-1} s^{-1} \phi(s), \quad(s>0) .
$$

Hence, it follows from (3.2) that

$$
\int_{X} \phi\left(M_{P} f\right) d \mu \leq \alpha \beta(\beta-1)^{-1} \int_{X} \phi(|f|) d \mu \quad\left(f \in L_{1} \cap L_{\phi}\right),
$$

where $\alpha$ is a constant in the $\triangle_{2}$-condition for $\phi$.

Since $|\hat{T} f| \leq P|f|$ for $f \in L_{1},(3.4)$ shows that there exists a constant $C_{1}>0$ such that $f_{X} \phi\left(M_{T} f\right) d \mu \leq C_{1} \int_{X} \phi(\mid f i) d \mu, f \in L_{1} \cap L_{\phi}$, which proves that $\left\|M_{T} f\right\|_{(\phi)} \leq C\|f\|_{(\phi)}, f \in L_{1} \cap L_{\phi}$, where $C=\max \left(1, C_{1}\right)$. Since $L_{1} \cap L_{\phi}$ is a dense linear subspace of $\left[L_{\phi},\|\|_{(\phi)}\right]$ it follows that $\left\|M_{T} f\right\|_{(\phi)} \leq C\|f\|_{(\phi)}$ for every $f \in L_{\phi}$, which proves a).

Now, let $\left\{b_{k}\right\}$ be a bounded Besicovitch sequence; then a) and the Banach principle show that for almost everywhere convergence it is enough to prove that the weighted averages

$$
T_{n} f=\frac{1}{n} \sum_{k=0}^{n-1} b_{k} T^{k} f
$$

converges a.e. for all $f$ in a dense subset of $\left[L_{\phi},\|\|_{(\phi)}\right]$.

Let $m \in N$ and $S: L_{\phi} \rightarrow L_{\phi}$ defined by $S f=e^{\mathrm{i} m} T f$. Since $L_{\phi}$ is reflexive and the powers $S^{k}, k \geq 0$, are uniformly bounded, exactiy $\left\|S^{k} f\right\|_{(\phi)} \leq\|f\|_{(\phi)}$ for every $f \in L_{\phi}$ and $k \geq 0$, then, the Césàro averages $R_{n} f=n^{-1}(f+S f+$ $\ldots S^{n-1}$ ) converge in norm for every $f \in L_{\phi}$. Therefore $L_{\phi}$ is the closure of 
the direct sum of the set of fixed points of $S$ and the space $(I-S) L_{\phi}$ (see 2.1 in $[6])$.

On the other hand, given $\beta>1$ such that $\beta \phi(s) \leq s \varphi(s), s \geq 0$, the function $s \longrightarrow s^{-\beta} \phi(s)$ increases for $s>0$ and consequently $\phi(s t) \leq s^{\beta} \phi(t)$ for $0 \leq s \leq 1$ and $t \geq 0$. Therefore, if $g \in L_{\phi}$ we have

$$
\begin{aligned}
\int_{X} \sum_{n=1}^{\infty} \phi\left(\left|n^{-1} S^{n} g\right|\right) d \mu \leq \sum_{n=1}^{\infty} n^{-\beta} \int_{X} \phi\left(\left|S^{n} g\right|\right) d \mu & \leq \\
& \leq \int_{X} \phi(|g|) d \mu \sum_{n=1}^{\infty} n^{-\beta}<\infty
\end{aligned}
$$

Hence $n^{-1} S^{n} g(x) \longrightarrow 0$ a.e. as $n \longrightarrow \infty$ and thus $R_{n} f \longrightarrow 0$ a.e. if $f=g-S g$.

Since the maximal operator $M_{S}$ is bounded in $\left[L_{\phi},\|\|_{(\phi)}\right]$ we obtain that, for any $f \in L_{\phi}, n^{-1} \sum_{k=0}^{n-1} e^{i m k} T^{k} f$ converges a.e. and therefore for every trigonometric polynomial $\alpha$ and $f \in L_{\phi}$ we have that

$$
\lim _{n \rightarrow \infty} \frac{1}{n} \sum_{k=0}^{n-1} \alpha(k) T^{k} f(x)
$$

exists and is finite a.e.

Then, for every $f \in L_{\phi} \cap L_{\infty}, T_{n} f$ converges a.e. since for every $\varepsilon>0$ there exists a trigonometric polynomial $\alpha_{\varepsilon}$ such that

$$
\limsup _{n \rightarrow \infty} \frac{i}{n} \sum_{k=0}^{n-1}\left|b_{k}-\alpha_{\varepsilon}(k)\right|<\varepsilon
$$

and consequentiy

$$
\limsup _{n \rightarrow \infty}\left|T_{n} f(x)-\frac{1}{n} \sum_{k=0}^{n-1} \alpha_{\varepsilon}(k) T^{k} f(x)\right|<\varepsilon\|f\|_{\infty} \quad \text { a.e. }
$$

In this way, since $L_{\phi} \cap L_{\infty}$ is dense in $L_{\phi}$, we conclude that $T_{n} f$ converges almost everywhere for every $f \in L_{\phi}$.

Finally, let $f^{*}(x)=\lim _{n \rightarrow \infty} T_{n} f(x)$. It follows from a) that $f^{*} \in L_{\phi}$ and $\phi\left(\left|T_{n} f-f^{*}\right|\right)$ is dominated by $\phi\left(M_{T} f\right) \in L_{1}$; thus, taking into account the Lebesgue's dominated theorem, we get that $\lim _{n \rightarrow \infty} \int_{X} \phi\left(\left|T_{n} f-f^{*}\right|\right) d \mu=0$ which proves that $\lim _{n \rightarrow \infty}\left\|T_{n} f-f^{*}\right\|_{(\phi)}=0$.

Acknowledgments.- This paper contains some of the resuits of the author's Doctoral Thesis written under the direction of Professor Alberto de la Torre at the University of Málaga. The author is deeply indebted to Professor de la Torre for his generous help. 


\title{
References
}

1. M.A. AKCOGLU AND R.V. ChaCón, A convexity theorem for positive operators, $Z W 3$ (1965), 328-332.

2. B. BRU ET H. HEINICH, Isométries positives et propriétés ergodiques de quelques espaces de Banach, Ann. Ins. Henri Poincaré 17 (1981), 377-405.

3. F. Cunningham and N. Grossman, On Young's inequality, Amer. Math. Monthly 78 (1971), 781-788.

4. A. GARSIA, Topics in almost everywhere convergence, Lectures in advances Math. Mark. Publ. Co. (1970).

5. M.A. KRasnoselsky AND V.B. Rutitsky, Convex functions and Orlicz spaces, Noordhoff Groningen (1961).

6. U. KRENGEL, Ergodic Theorems, W. de Gruyter. Studies in Mathematics (1985).

7. J. MUSIELAK, Orlicz spaces and modular spaces, "Springer-Verlag," 1983.

8. J.H. OLSEN, The individual weighted ergodic theorem for bounded Besicovitch sequences, Canad. Math. Bull. 25 (1982), 468-471.

9. R. SATO, An extrapolation theorem for contractions with fixed points, Canad. Math. Bull. 24 (1981), 199-203.

10. A. DE LA TORRE, A dominated ergodic theorem for contractions with fixed points, Canad. Math. Bull. 20 (1977), 89-91.

\author{
Departamento de Matemáticas \\ Facultad de Ciencias \\ Universidad de Málaga \\ SPAIN
}

Rebut el 8 d'octubre de 1987 